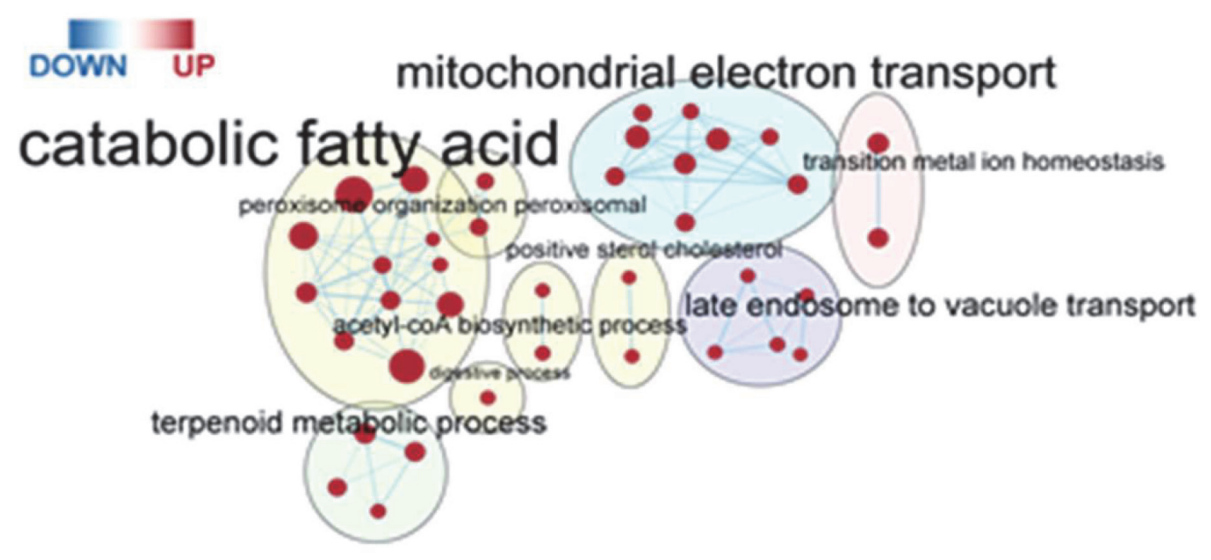

Abstract P051 Figure 1 Pathway analysis with Gene Set Enrichment Analysis (GSEA) in mice hepatocytes overexpressing miR29b compared to controls. Lipid metabolism pathways shown with a yellow background were predominantly enriched

were predominately upregulated by miR29b (figure 1). Systems-level analysis revealed that miR-29b levels were significantly decreased during the pathogenetic stages from liver steatosis to fibrosis. The reduced levels of miRNA29b were associated with lipid accumulation and inflammation in the liver. Further investigation revealed that miR29b degraded Insulin Receptor Substrate 1(IRS1) via directly targeting its 3'UTR. Moreover, mice injected with miR29b micelles alleviated hepatic lipid accumulation via downregulating fatty acid synthesis through IRS1-related pathway and enhanced $\beta$ oxidation.

We report that lipid metabolism-related pathways were significantly upregulated by miR29b. MiR29b ameliorated hepatic lipid accumulation via directly targeting IRS1-regulated lipid metabolism. In addition, the analysis of other impacted biological pathways and the networks involving miR29b and hepatic-associated mRNAs or non-coding RNAs is in progress.

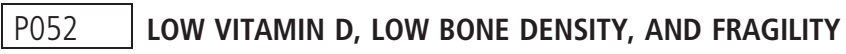 FRACTURES ARE COMMON AMONG UK LIVER TRANSPLANT RECIPIENTS}

Georgeina L Jarman*, Gwilym J Webb. Cambridge Liver Unit, Cambridge University Hospitals NHS Foundation Trust, Addenbrooke's Hospital, Cambridge, CB2 OQQ, UK

\subsection{6/gutjnl-2021-BASL.61}

Introduction Patients with chronic liver disease are at risk of osteodystrophy, with increased fracture risk post-transplantation. National guidelines recommend all patients with chronic liver disease are offered calcium and vitamin D supplementation and bone mineral density (BMD) assessment, usually through DEXA (Dual energy X-Ray Absorptiometry) imaging. Guidelines advise testosterone measurement in men with reduced BMD. There is a paucity of evidence surrounding Vitamin D deficiency and fragility prevalence in liver transplant recipients.

Methods Liver transplant recipients over 3 years to 2021-0512 were evaluated, with super-urgent listings excluded. Retrospective analysis of electronic hospital records of transplant assessment was conducted. Records were examined for demographics, total vitamin D $(25(\mathrm{OH}) \mathrm{D} 2+25(\mathrm{OH}) \mathrm{D} 3)$, testosterone levels within 6 months, DEXA imaging within 2 years, prescription of bone-protective medications, and diagnoses of fragility fractures.

Results Of 312 liver transplants, 29 super-urgent listings were excluded and 283 assessed. Median age at transplant assessment was 56 years (IQR 48-63), 180 (64\%) of recipients were male, and 262 (93\%) had received a first graft.

DEXA diagnoses of reduced BMD (osteopaenia/osteoporosis) were present in $36(13 \%)$ at transplant assessment. 118 (42\%) underwent DEXA imaging within 2 years, with COVID-19 reducing scan availability. Of those who underwent DEXA imaging, 56 (64\%) received a diagnosis of osteopaenia and $20(23 \%)$ osteoporosis. Among men with reduced BMD, only 2 of 46 (4\%) underwent testosterone testing within 6 months, both with normal testosterone.

Total vitamin D tested within 6 months was measured in 274 (97\%). 138 (49\%) were deficient (<30nmol/L) and $75(27 \%)$ borderline $(30-50 \mathrm{nmol} / \mathrm{L})$. Median total vitamin $\mathrm{D}$ of the cohort was $30 \mathrm{nmol} / \mathrm{L}(18-48)$. Of those (157, $55 \%)$ not receiving supplementation within 6 months prior to assessment, median vitamin $\mathrm{D}$ was $26 \mathrm{nmol} / \mathrm{L}(15-37)$ with $59 \%$ deficient; total vitamin D was higher at $38 \mathrm{nmol} /$ L (22-58) in those on supplementation, with $37 \%$ deficient $(\mathrm{p}<0.001)$.

Fragility fractures had occurred prior to transplantation in 32 (11\%). Recipients were followed for a median of 621 days post-transplant (267-877). 26 (9\%) sustained new fragility fractures over the course of follow up. Fragility fractures

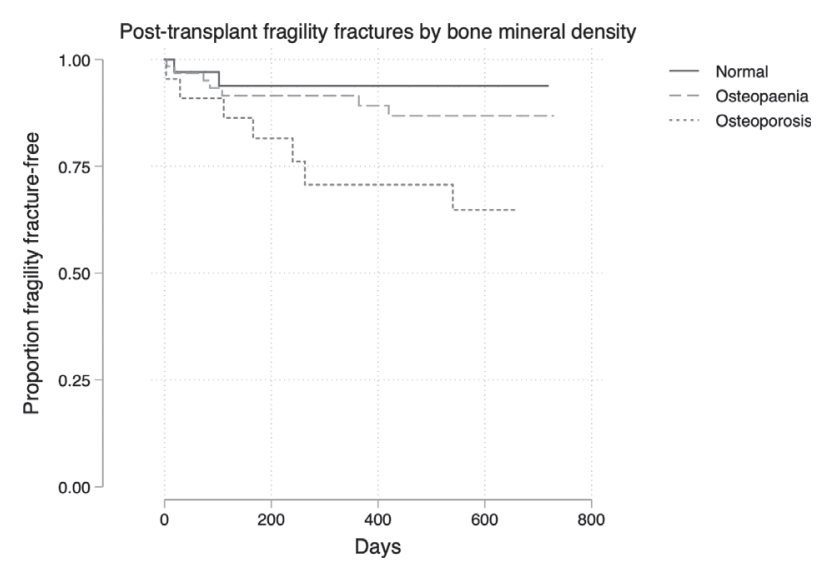

Abstract P052 Figure 1 
correlated with reduced bone density and female sex, but neither vitamin D status nor pre-transplant fragility fractures.

Discussion Liver transplant recipients are commonly deficient in Vitamin D, and at high risk of reduced bone density and fragility fractures both pre- and post-transplantation. Further evaluation of factors predisposing recipients to fragility fractures, and adherence to national and local guidelines on optimising bone health, are required.

\section{P053 INVESTIGATION OF THE ORIGIN OF SERUM NEUTROPHIL GELATINASE ASSOCIATED LIPOCALIN (NGAL) FOLLOWING LIVER ISCHAEMIA REPERFUSION AND RESULTANT ACUTE KIDNEY INJURY IN MICE}

Esther Platt*, Francis Robertson, Andrew Hall, Alberto Quaglia, Brian Davidson. University College London, London, UK

\subsection{6/gutjnl-2021-BASL.62}

Ischaemia reperfusion (IR) injury to the liver is independently associated with development of acute kidney injury in patients following liver transplantation. Neutrophil Gelatinase Associated Lipocalin (NGAL) is an early marker of primary Acute Kidney Injury (AKI) in mice and humans. Using a mouse model of remote ischaemic preconditioning, we investigated whether serum NGAL concentration correlates with development of AKI following ischaemia reperfusion of the liver and the origin of serum NGAL in this setting.

$24 \mathrm{C} 56 \mathrm{BL} 6 / \mathrm{J}$ mice were divided into 4 groups (6 mice per group): a) sham b) liver ischaemia reperfusion (IR) c) remote ischaemic preconditioning (RIPC) d) RIPC + IR.

RIPC was achieved by interval clamping of the right femoral vessels with sham mice undergoing vessel exposure without clamping. Subsequent partial liver ischaemia was achieved by cross-clamping the inflow to left and middle liver lobes for 45 minutes followed by 2 hours of reperfusion. Sham mice underwent laparotomy without clamping. Plasma samples were collected by exsanguination at the end of 2 hours reperfusion and the liver and left kidney were harvested, fixed in formalin and paraffin embedded. Plasma transaminase levels were used as a surrogate marker of liver IR injury. Serum creatinine was used as a marker of AKI and NGAL levels were also performed. Tissue specimens were processed for quantitative PCR, NGAL immunohistochemistry and NGAL/F4/80 dual staining.
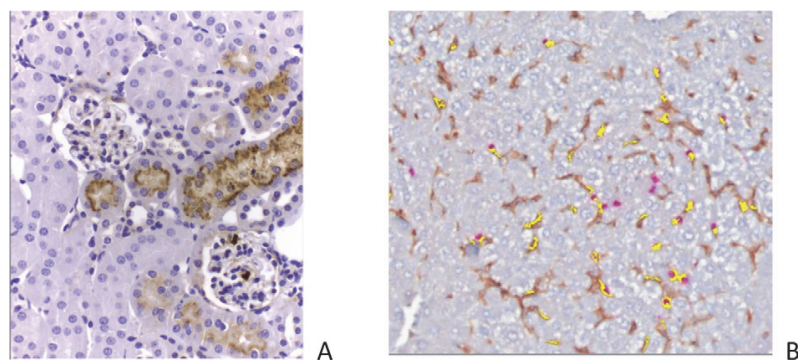

Abstract P053 Figure 1 NGAL immunohistochemistry (IHC) of mouse kidney specimen in ' $A$ ' demonstrates staining within the apical third of tubular cells, indicating possible uptake of NGAL from tubular filtrate. This is in keeping with established literature. 'B' demonstrates dual staining of $F 4 / 80$ positive cells (brown stain) and NGAL (pink stain) in a mouse liver specimen. Co-localisation of F4/80 and NGAL is depicted by yellow marker. This indicates partial NGAL expression by F4/80 (Kupffer) cells, with no hepatocyte NGAL expression. As depicted in this slide, at least one other cell type expresses NGAL within the liver
Comparing mice in group b) with the other three groups, serum transaminase levels correlated with both serum creatinine and serum NGAL, indicating that liver IR is significantly associated with liver injury, development of AKI and an increase in serum NGAL concentration in mice.

Quantitative PCR identified no difference in NGAL RNA expression between the groups. This was supported by NGAL immunohistochemistry findings. Liver and kidney specimens demonstrated no difference in the number of NGAL positive cells or intensity of NGAL staining between different groups of mice (figure 1). In the kidney, NGAL staining within the apical third of tubular cells suggested uptake of NGAL from tubular filtrate. In the liver, NGAL staining spared hepatocytes but demonstrated partial co-localisation with F4/80, a marker for Kupffer cells.

We demonstrated an association between liver IR injury, AKI and serum NGAL that is present only 2 hours post reperfusion in mice. Interestingly NGAL does not appear to originate from either the liver or the kidney within this time frame, in contrast to other published data indicating production of NGAL by hepatocytes at later time points.

\section{P054 LONG TERM SURVIVAL AND HEALTHCARE RESOURCE USE IN PATIENTS WITH HEPATIC ENCEPHALOPATHY RECEIVING RIFAXIMIN- $\alpha$ TREATMENT: A RETROSPECTIVE OBSERVATIONAL EXTENSION STUDY WITH LONG TERM FOLLOW-UP (IMPRESS II)}

\begin{abstract}
${ }^{1}$ Mark Hudson* ${ }^{2,3}$ Stephen D Ryder, ${ }^{4}$ Paul Richardson, ${ }^{5}$ Debbie L Shawcross, ${ }^{6}$ Elizabeth Farrington, ${ }^{7}$ Mark Wright, ${ }^{8}$ Robert T Przemioslo, ${ }^{9}$ F Porras Perez, ${ }^{9}$ Melanie Kent, ${ }^{10}$ Bharat Amlani, ${ }^{11}$ Joe Hickey, ${ }^{12}$ Richard Aspinall. 'Liver Unit, Freeman hospital, Newcastle upon Tyne, UK; ${ }^{2}$ NIHR Biomedical Research Unit in Gastrointestinal and Liver Diseases at Nottingham University Hospitals NHS Trust, Nottingham, UK; ${ }^{3}$ The University of Nottingham, Nottingham, UK; ${ }^{4}$ Department of Gastroenterology and Hepatology, Royal Liverpool and Broadgreen University Hospitals NHS Trust, Liverpool, UK; ${ }^{5}$ Institute of Liver Studies and Transplantation, King's College London School of Medicine at King's College Hospital, London, UK; ${ }^{6}$ Department of Gastroenterology and Hepatology, Royal Cornwall Hospital, Cornwall, UK; ${ }^{7}$ Department of Hepatology, University Hospital Southampton, Southampton, UK; ${ }^{8}$ Department of Gastroenterology, Southmead Hospital, Bristol, UK; ${ }^{9}$ Department of Gastroenterology, University Hospital of North Durham, Durham, UK; ${ }^{10}$ Global Medical Affairs, Norgine, Harefield, UK; ${ }^{11}$ OPEN Health, The Weighbridge, Brewery Courtyard, High Street, Marlow, UK; ${ }^{12}$ Department of Gastroenterology and Hepatology, Queen Alexandra Hospital, Portsmouth, UK
\end{abstract}

\subsection{6/gutjnl-2021-BASL.63}

Hepatic encephalopathy (HE) is associated with high mortality and considerable resource use including recurrent hospital admissions in people with cirrhosis. IMPRESS was a 12-month observational study of 207 patients with HE, demonstrating addition of rifaximin- $\alpha$ led to reduced hospitalisations, Emergency Department attendances and 30-day readmissions. ${ }^{1}$ Data on long-term (1-5 year) outcomes with rifaximin- $\alpha$ use is scarce. An extension to IMPRESS was conducted to evaluate outcomes up to 5 years (IMPRESS II).

Data were obtained from medical records of 138 patients from 9/13 centres included in IMPRESS, including demographic, clinicopathological, treatment and resource use for the period from rifaximin $-\alpha$ initiation (index) to 5 years post-index or death. Long-term survival is reported herein.

Overall, 62\% (85/138) of patients were male; mean (standard deviation [SD]) age at cirrhosis diagnosis, HE diagnosis and index was $58.2(12.1[\mathrm{n}=133]), 60.2(11.7)$, and 60.9 (11.6), respectively. At index, 9\%, 50\% and 41\% of patients $(\mathrm{n}=127)$ had albumin-bilirubin (ALBI) grade I, II and III, 